\title{
The Relationships between Fitness Assessments, Fitness Levels and Coronary Heart Disease Risk Markers in Police Officers
}

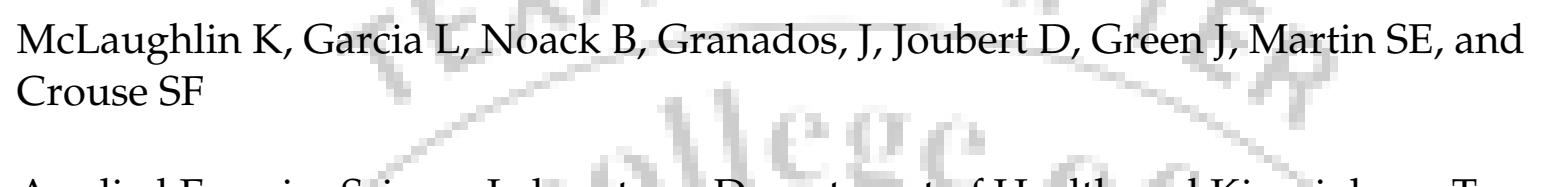

Applied Exercise Science Laboratory; Department of Health and Kinesiology; Texas A\&M University; College Station, TX

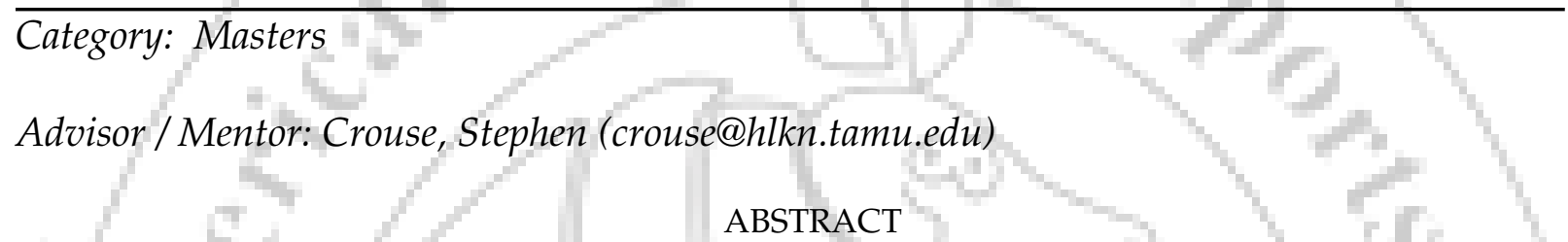

Given the physical nature of a position in law enforcement, the assessment of cardiovascular (CV) health as well as muscular strength and endurance is paramount to ensuring the safety of these personnel and the citizens they serve. The present study retrospectively examined correlations between several markers of $\mathrm{CV}$ and muscular fitness for 65 local police officers ( 8 female, average age $35.6 \pm 9.0 \mathrm{yrs}$, height $70.5 \pm 2.8$ in, weight $91.8 \pm 18.7 \mathrm{~kg}, \mathrm{VO}_{2} 33.0 \pm 5.3 \mathrm{~mL} / \mathrm{kg} / \mathrm{min}$ ) taken from their annual screening including a graded exercise test (GXT; Bruce protocol), pushups completed in 1 minute, situps completed in 1 minute, handgrip strength, body composition (through DEXA and circumference measures) and bloodwork. Maximal oxygen uptake $\left(\mathrm{VO}_{2 \max }\right)$ was estimated using the Foster equation. Coronary heart disease risk (CHD risk) was calculated using the online NIH 10-year heart attack risk calculator, which uses age, gender, total cholesterol, HDL cholesterol, smoking status, and blood pressure status in its algorithm. Significance was determined using an alpha level of 0.05 . Interestingly, there was no significant correlation between $\mathrm{VO}_{2 \max }$ and $\mathrm{CHD}$ risk $(\mathrm{p}=0.1851)$. Apart from risk markers included in the risk calculation, $\mathrm{CHD}$ risk was significantly correlated with waist-hip ratio $(r=0.24739)$, and LDL cholesterol $(r=0.39117)$. $\mathrm{VO}_{2 \max }$ was significantly negatively correlated with fat mass $(\mathrm{r}=-0.51849)$, body fat percentage $(\% B F ; r=-0.64297)$, total cholesterol $(T C ; r=-0.3371)$, and LDL cholesterol $(r=-0.267)$. Positive correlations were found between $\mathrm{VO}_{2 \max }$ and other measures of fitness such as pushups $(\mathrm{r}=0.54274)$, situps $(\mathrm{r}=$ $0.3282)$, and handgrip strength $(\mathrm{r}=0.32041)$. As for body composition measures, $\% \mathrm{BF}$ was significantly positively correlated with TC $(r=0.30188)$, systolic blood pressure $(r=0.28795)$, LDL cholesterol $(r=$ $0.26533)$, and fasting glucose $(r=0.26536)$, but negatively correlated with fitness markers such as pushups $(\mathrm{r}=-0.58599)$, situps $(\mathrm{r}=-0.43604)$, and handgrip strength $(\mathrm{r}=-0.32393)$. Lean mass was significantly correlated with handgrip strength $(\mathrm{r}=0.71835)$, though not pushups, situps or $\mathrm{VO}_{2 \max }$. Waist circumference was significantly negatively correlated with HDL cholesterol $(r=-0.39202)$. Additionally, 62 of the participants ( 7 female) completed both a 1.5 mile run and GXT, allowing for a comparison of a submaximal to a maximal fitness assessment of CV fitness. Time to complete 1.5 miles was significantly correlated to GXT VO $\mathrm{VO}_{2 \mathrm{max}}$, with a Pearson correlation coefficient of -0.79593 . Overall, relationships found between health markers, fitness, and CHD risk in this sample were in line with previous research on CHD risk factors and the assessment of $\mathrm{CV}$ and muscular fitness. 